\title{
Influence of Atmospheric Conditions on mmWave CATR Facilities: Formulation and Compensation Scheme
}

\author{
A. Muñoz-Acevedo ${ }^{1}$, L. Rolo ${ }^{2}$, M. Sierra-Castañer ${ }^{3}$, M. Paquay ${ }^{4}$ \\ ${ }^{1}$ Radiation Group, Technical University of Madrid (UPM), Madrid, Spain, alfonso@gr.ssr.upm.es \\ ${ }^{2}$ Antenna and Sub-Millimetre Wave Section, European Space Agency, Noordwijk, The Netherlands, 1.rolo@esa.int \\ ${ }^{3}$ Radiation Group, Technical University of Madrid (UPM), Madrid, Spain, mscastaner@gr.ssr.upm.es \\ ${ }^{4}$ Antenna and Sub-Millimetre Wave Section, European Space Agency, Noordwijk, The Netherlands, m.paquay@esa.int
}

\begin{abstract}
The performance of a CATR relies on the planarity of the synthesized test wave, which is generated within a bounded volume for which specifications are drawn. Millimetre-wave facilities deal with the classical limitations of this frequency band, among which two become critical in our analysis: time-extensive acquisition campaigns and impact of environmental variables. Both features become more evident when increasing the frequency of operation. The variation in atmospheric variables, such as humidity, temperature and pressure has an influence over the performance of all the elements of the facility. The instrumentation behavior is influenced both by the warming up process, and the ambience conditions that surround the equipment. On the changes of the atmosphere itself, they affect the electromagnetic wave propagation, given the physical link between the conditions of the atmosphere and its electric properties as an electromagnetic waves propagation medium.
\end{abstract}

Keywords: CATR, millimetre wavelength, range assessment, quiet zone probing.

\section{INTRODUCTION}

Antenna measurement ranges are facilities capable to measure the radiation pattern of antennas under test. Compact Antenna Test Ranges (CATRs) are a particular kind of measurement facility which performs measurements in farfield conditions within a reduced, indoor chamber [1]. This compactness is ensured through well studied electromagnetic field collimating schemes, for which diverse implementations have proven to develop test performance [2].

CATR facilities offer indoor conditions where environmental variables keep stable within certain bounds. At mm-wavelengths, these environmental specifications may not ensure electrical performance. This is due to two physical evidences: the environmental variability of the atmosphere and the well known sensitivity of mm-wavelength frequencies to these changes. This scenario derives in facilities whose electrical behavior is environmentally dependent, affecting the ability of the facility to perform antenna characterization.

Most critical is, however, the fact that range assessment processes itself are the most sensitive to this environmental dependence, in virtue of their intrinsically extensive temporal extension, which enlarges the impact window of the environmental drifts [3]. Regarding quiet zone probing, ambience drifts act aggressively over the phase of the sampled test wave. It masks phase patterns that can be used as monitors of the range's performance in terms of feed focusing, collimators alignment, or their electromagnetic performance. Consequently, environmental drift and its impact over the electrical performance of mm-wavelength CATRs makes the time-costly assessment results hard to handle, or simply useless if its impact is obviated as an influent factor.

This drift in electrical performance has been studied in the past with the purpose of performing a correction as accurate as possible. Traditionally, focus has been set either over the cables' electrical performance or in the instrumentation drift [4,5], with diverse compensation scheme implementations. This paper contributes the literature performing analysis and correction in the radiating stage of the CATR, through its collimation optics. Our focus is set here parting from the experimental evidence that the propagation path the most environmentally-sensitive stage [3], for our CATR of interest.

\section{ELECTRICAL BEHAVIOR OF THE ATMOSPHERE}

\section{A. Physical model}

A general case studies the evolution of both the real and imaginary parts of the propagation path's permittivity $\varepsilon$ and permeability $\mu$. That analysis allows the seek for resonant behavior of the atmosphere, with potential use at frequencies beyond $100 \mathrm{GHz}$, as it is of interest in [6]. Characterization of both real and imaginary parts for $\varepsilon, \mu$ enables accurate evaluation of magnitude-phase response through the propagation path.

The model proposed here focuses on the behavior of the permittivity's real part $\varepsilon^{\prime}=\operatorname{Re}(\varepsilon)$, responsible for the phase 
shift $\Delta \phi$ along the path between feed and probe $\Delta r$, as in Eq. 1. Magnetic permeability is assumed with confidence to be real and constant $\mu=\mu_{0}$. The imaginary part of $\varepsilon$ is not considered, given that it is mainly responsible for the attenuation and molecular resonance phenomena, which are not of our interest in this communication and mainly apply at frequencies above $100 \mathrm{GHz}$ [6].

$\Delta \phi(\boldsymbol{\Gamma})=k_{0} \cdot \sqrt{\frac{\varepsilon^{\prime}(\boldsymbol{\Gamma})}{\varepsilon_{0}}} \cdot \Delta r$

The dependence of $\varepsilon^{\prime}$ with the environmental vector $\boldsymbol{\Gamma}$ is translated to the induced phase shift. As pointed out in $[7,8]$, $\Gamma$ can be reduced three components: temperature $T$, relative humidity $r h$ and atmospheric pressure $p_{\text {atm }}$. Accordingly, time dependent environment $\Gamma(t)$ requires sampling of those three variables, potentially with three logging devices. However, this scheme can be simplified by deducing the atmospheric pressure $p_{a t m}$ as a function of $T, r h$ if the (constant) altitude over sea level $h_{S L}$ is known and psychrometric calculations are considered [9,10]. It avoids the barometric logger, reducing the variables to be sampled to: $T, r h$. Full details on the $p_{\text {atm }}$ retrieval process are explained in Section III.

Eq. 2 models electrically the time-varying environment. The evolution of the refractive index $\sqrt{\varepsilon^{\prime}(\boldsymbol{\Gamma}) / \varepsilon_{0}}$ with the environment follows Eq. 3, being $e_{S}(T)$ the saturated water vapor pressure [11].

$$
\begin{aligned}
& \boldsymbol{\Gamma}(t)=\left[T(t), r h(t), p_{\text {atm }}\left(T(t), r h(t), h_{S L}\right)\right] \\
& \sqrt{\varepsilon^{\prime}(\boldsymbol{\Gamma}) / \varepsilon_{0}}=1+\frac{77.6}{T} \cdot\left[p_{a t m}\left(T, r h, h_{S L}\right)+4810 \frac{r h}{T \cdot 100} \cdot e_{s}(T)\right] \cdot 10^{-6}
\end{aligned}
$$

Calculation of $\varepsilon_{R}=\varepsilon^{\prime}(\boldsymbol{\Gamma}) / \varepsilon_{0}$ for CATR-ESTEC $\left(h_{S L}=30 m\right)$ is shown in Fig. 1. At the frequency of interest $106 \mathrm{GHz}$, it induces the phase drift pattern depicted in Fig. 2, as a function of $T, r h$ for a feed-probe path length $\Delta r=16 \mathrm{~m}$, respect to the homogeneous atmosphere-insensitive scenario $\varepsilon_{R}=1$.

\section{B. Correction scheme}

In this paragraph, it is presented a setup able to acquire and implement the data correction scheme. Fig. 3 depicts a systemlevel layout which summarizes relevant modules in this correction scheme. External mix is assumed for the mmwavelength signal generation, and field collimation mechanism is left open for sake of generality. The ambience sensor is a logging device inside the chamber able to sample relative humidity and temperature in a synchronized fashion. With the calculations of paragraph II.A, atmospheric phase drift $\Delta \phi$ is deduced, for each sampling location $\mathbf{r}_{A C Q}$.

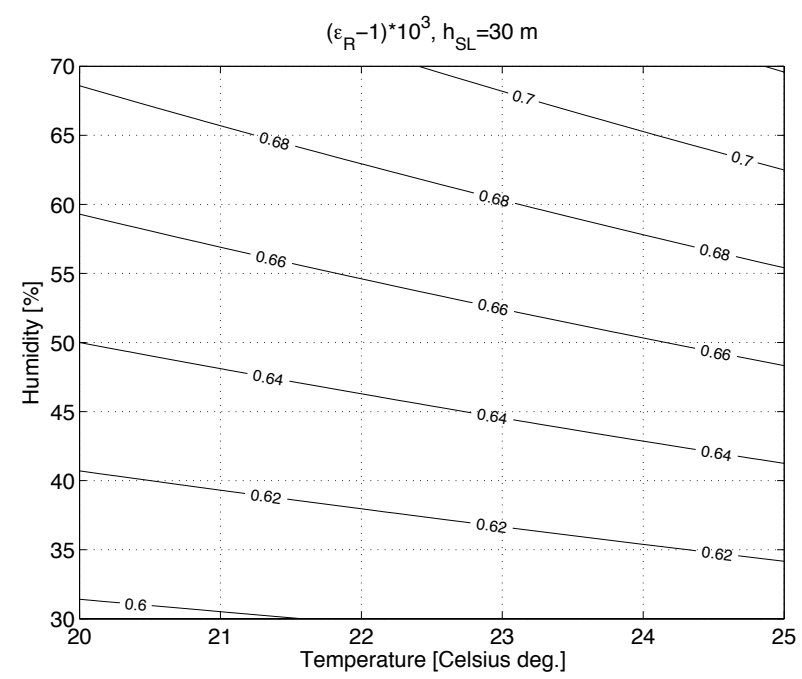

Fig. 1. Relative permittivity evolution for CATR-ESTEC

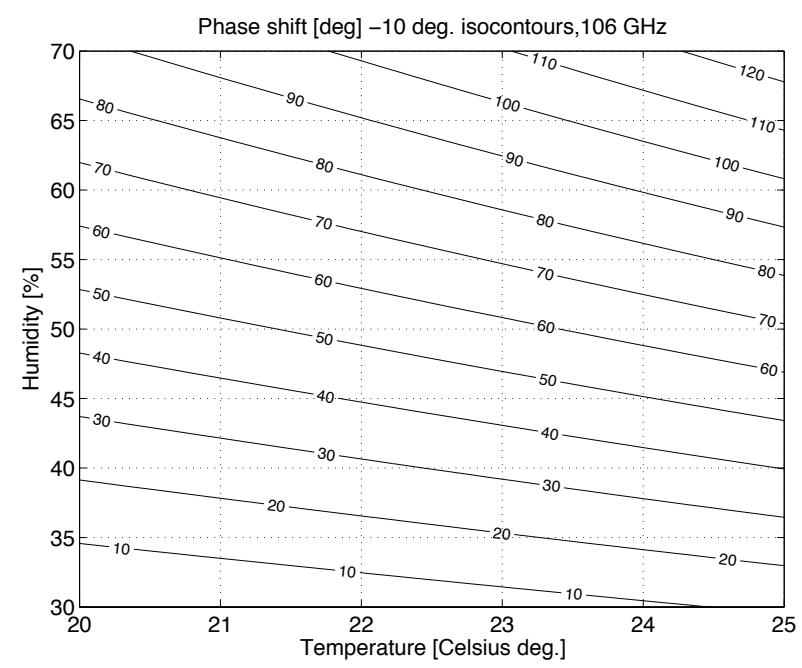

Fig. 2. Phase drift induced by atmospheric fluctuations (106 GHz)

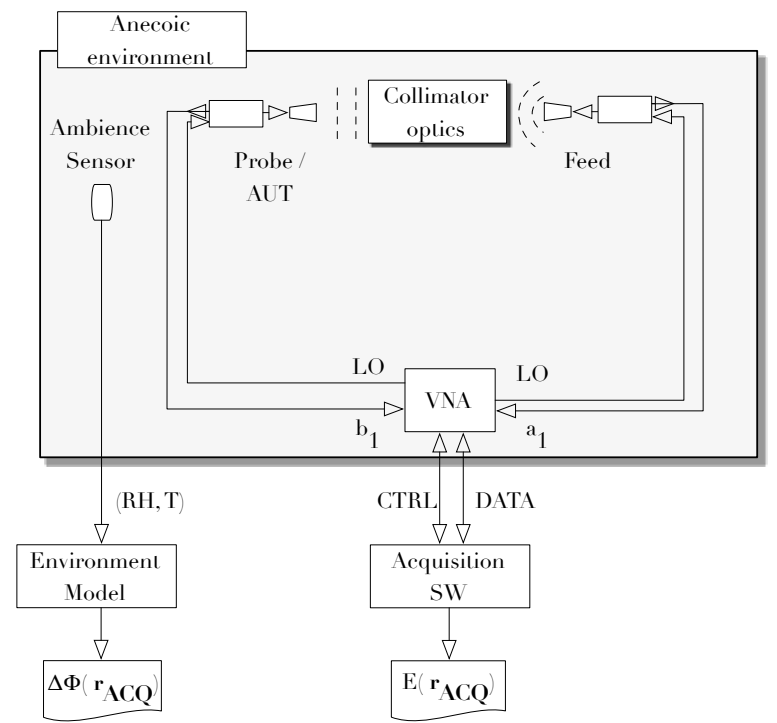

Fig. 3. Setup for environmental correction in CATR 
The nature of $\mathbf{r}_{A C Q}$ may vary depending on the kind of acquisition performed. If AUT characterization is carried out, $\mathbf{r}_{A C Q}$ potentially is a set of angular directions of interest. When quiet zone assessment tasks are performed, the sampler antenna is a probe and $\mathbf{r}_{A C Q}$ will move within the test region to be evaluated, potentially a transversal planar domain bounded either by a circle or a rectangle depending on the probing setup.

For this second scenario, environmental compensation may be complimented by probe compensation [12] over time-gated data, as proposed by the data post-processing scheme in Fig. 4.

\section{ATMOSPHERIC PRESSURE RETRIEVAL SCHEME}

The atmosphere is a mixture of diverse gaseous species which keep certain proportions studied by Chemistry of atmosphere. The vast majority of the atmospheric gases mantain fix the volumetric proportions among the different atmospheric layers [9]. However, unlike most of the gases, the proportion of water vapor in the atmosphere is strongly variable over space and time. Our analysis of a moisted atmosphere is based on the principle that the it is a mixture of two main species: dry atmosphere and water vapor. For each one of those, the ideal gases' equation of state (Eq. 4) applies, as well as it does for the moisted atmosphere (Eq. 5).

$$
\begin{aligned}
& p \cdot V=n_{m o l} \cdot R \cdot T \Leftrightarrow p=\rho \cdot \frac{R}{M} \cdot T \\
& p_{a t m}=\rho_{a t m} \cdot R_{a t m}(r, \xi) \cdot T
\end{aligned}
$$

Use of Eq. 4 for each one of the three species requires the introduction of the thermodynamic variables which are required for each of them. Table I summarizes these variables both for the two blending gases and the moisted atmosphere. Thus, the atmosphere satisfies Eq. 5, where $\operatorname{Ratm}(r, \xi)$ is not constant [Seinfeld 1998] but depends on:

- The mixture ratio $\mathrm{r}$ : is defined as the mass fraction between water vapor and dry air. It equals the densities' ratio, as in Eq. 11.

- The mixing species' constants ratio $\xi=\mathrm{R}_{\mathrm{d}} / \mathrm{R}_{\mathrm{w}}=0.622$, which is not affected by environmental changes.

Thermodynamic analysis of the variables on Table I leads to Eqs. 6 -10. The dry air density $\rho_{d}$ depends on the altitude over sea level $h_{S L}$ and absolute temperature $T$, while the water vapor density $\rho_{w}(T, R H)$ is obtained through psychrometric calculations, as in [10]. The dry air pressure component $p_{d}$ results from the integration of the dry atmosphere's density $\rho_{d}$ from sea level towards the altitude where $p_{d}$ is of interest, simplifying as Eq. 7 . Assuming a linear temperature decrease (Eq. 8 [9]) through a tropospheric constant gradient $\nabla_{T}$ (Eq. 9), Eq. 7 develops as Eq. 10.

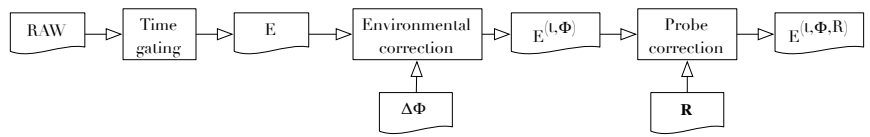

Fig. 4. Data post-processing scheme

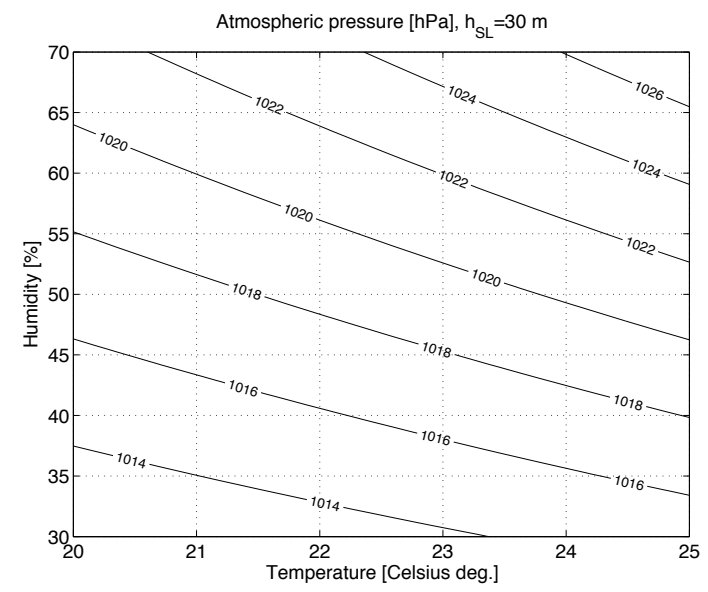

Fig. 5. Retrieved atmospheric pressure (hPa) at the location of experiment

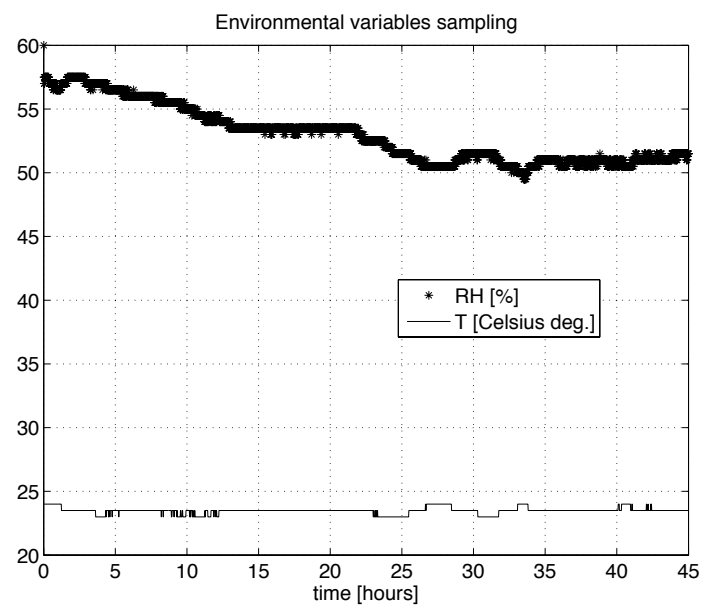

Fig. 6. Registered environmental variables

TABLE I. THERMODYNAMIC VARIABLES IN THE ATMOSPHERE

\begin{tabular}{|l|c|c|c|}
\hline \multirow{2}{*}{ Variable } & \multicolumn{2}{|c|}{ Blending gases } & \multirow{2}{*}{$\begin{array}{c}\text { Moisted } \\
\text { atmosphere }\end{array}$} \\
\cline { 2 - 3 } & $\begin{array}{c}\text { Dry } \\
\text { atmosphere }\end{array}$ & Water vapor & $p_{\text {atm }}$ \\
\hline Pressure & $p_{d}$ & $e$ & $\rho_{\text {atm }}\left(T, R H, h_{S L}\right)$ \\
\hline $\begin{array}{l}\text { Volumetric } \\
\text { mass density }\end{array}$ & $\rho_{d}\left(T, h_{S L}\right)$ & $\rho_{w}(T, R H)$ & $R_{\text {atm }}(r, \xi)$ \\
\hline $\begin{array}{l}\text { Species } \\
\text { constant }\end{array}$ & $R_{d}$ & $R_{w}$ & $T$ \\
\hline Temperature & $T$ & $T$ & $T$ \\
\hline
\end{tabular}




$$
\rho_{\text {atm }}\left(T, R H, h_{S L}\right)=\rho_{d}\left(T, h_{S L}\right)+\rho_{w}(T, R H)
$$

$p_{d}\left(T, h_{S L}\right)=p_{0} \cdot \exp \left(-\frac{g}{R_{d}} \cdot \int_{0}^{h_{S L}} \frac{1}{T(\tau)} d \tau\right)$

$T(\tau)=T\left(h_{S L}\right)+\left(\tau-h_{S L}\right) \cdot \nabla_{T}=\nabla_{T} \cdot\left(\tau-\left(h_{S L}+\frac{T\left(h_{S L}\right)}{\nabla_{T}}\right)\right)$

$\nabla_{T}=-6.63 \cdot 10^{-3} K \cdot m^{-1}$

$p_{d}\left(T, h_{S L}\right)=p_{0} \cdot \exp \left(-\frac{g}{R_{d} \cdot \nabla_{T}} \cdot \ln \left(\frac{\left|T\left(h_{S L}\right) / \nabla_{T}\right|}{\left|h_{S L}+T\left(h_{S L}\right) / \nabla_{T}\right|}\right)\right)$

The integration of the dry atmosphere's partial pressure $p_{d}$ into the total atmospheric pressure $p_{a t m}$ requires the knowledge of the water vapour's density $\rho_{w}$ and $R_{a t m}$, expressed as a function of the mixing ratio $r$ and the mixing species' constants ratio $\xi=R_{d} / R_{w}=0.622$ (Eqs. 11, 12). These equations lead to the expression of the atmospheric pressure $p_{a t m}$, as in Eq. 13 . Evolution of $p_{a t m}$, in $\mathrm{hPa}$, as a function of $(\mathrm{T}, \mathrm{RH})$ for a fix altitude over sea level hsL $=30 \mathrm{~m}$ can be found in Fig. 5 .

$$
\begin{aligned}
& r \equiv r\left(T, R H, h_{S L}\right)=\frac{\rho_{w}(T, R H)}{\rho_{d}\left(T, h_{S L}\right)} \\
& R_{a t m}=R_{a t m}(r, \xi)=R_{d} \cdot\left(\frac{1+r / \xi}{1+r}\right) \\
& p_{a t m} \equiv p_{a t m}\left(T, R H, h_{S L}\right)=\ldots
\end{aligned}
$$$$
\ldots=\left[\rho_{d}\left(T, h_{S L}\right)+\rho_{w}(T, R H)\right] \cdot R_{d}\left(\frac{1+r\left(T, R H, h_{S L}\right) / \xi}{1+r\left(T, R H, h_{S L}\right)}\right) \cdot T
$$

\section{NUMERICAL RESULTS}

Up to date, a $\arg \left\{S_{2,1}\right\}$ measurement was performed. Probe is set to fix position during extensive acquisition time and IFBW is set as low as $100 \mathrm{~Hz}$ in order to filter out the instrumentation noise. Acquired data is properly time-gated to remove stray contributions out of the direct ray's timeslot. Indoor humidity and temperature are sampled in a synchronized way, sharing the same time axis. Plots of these magnitudes are shown in Fig. 6, for which the retrieved atmospheric pressure is plotted in Fig. 7.

Using the evolution of the magnitudes depicted in Figs. 6, 7 together with a wrapped version of the phase in Eq. 1, the phase drift induced by atmosphere is calculated and plotted

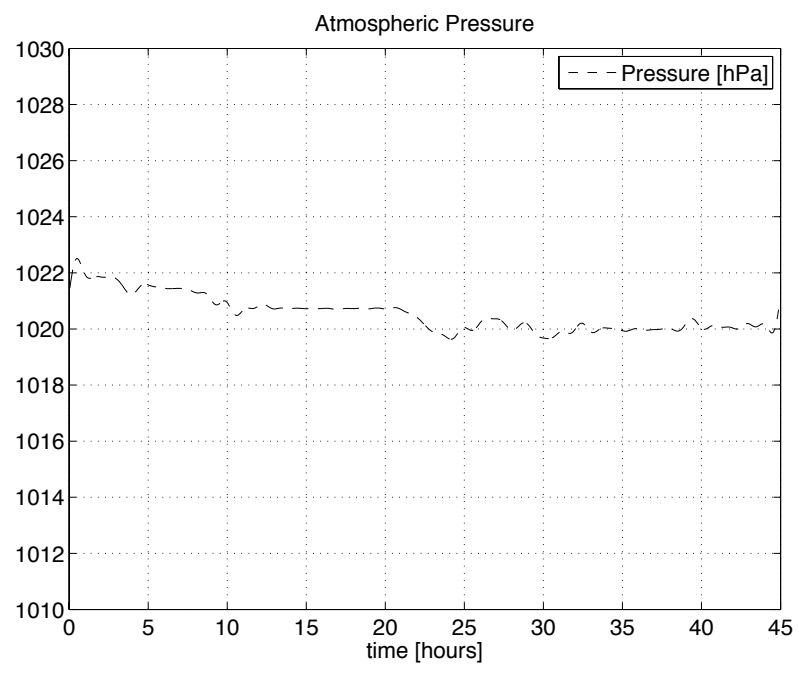

Fig. 7. Retrieved atmospheric pressure (hPa)

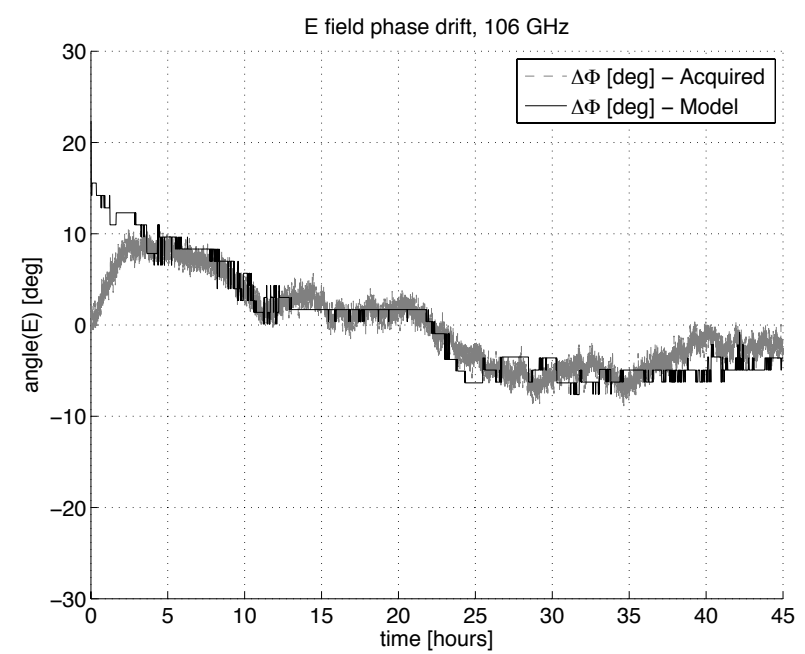

Fig. 8. Phase drift at 106 GHz. Breathing results vs. model.

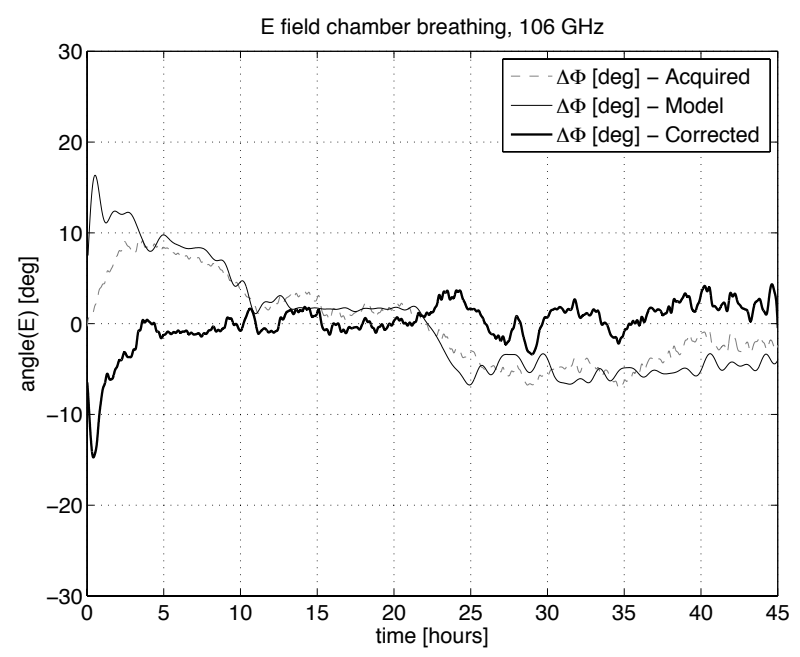

Fig. 9. Phase drift at $106 \mathrm{GHz}$ - with correction. 
together with the acquired phase in Fig. 8. Besides the phase wrapping, both plots are normalized removing their average value so valid comparison is possible.

Good agreement is found between the traces. The simplified model is able to draw the phase drift pattern of the long term acquisition experiment, over which many other factors have an influence that escapes from the scope of our approach. Potentially, the most remarkable of these factors is the instrumentation drift, which coherently is summed to the contribution of the atmosphere. The influence of this instrumentation drift should be more evident during the first hours of the experiment, when the instruments are heating up. While simple, this approach can be observed in a differential analysis of plots in Fig. 8: main discrepancy occurs within the first 3 hours, where the monotony of the phase plots is actually the opposite, being convergent beyond the $3^{\text {rd }}$ hour of experiment.

For sake of clarification, the two patterns are low pass filtered and phase compensation is performed following the scheme described in II.B. Joint plotting is performed in Fig. 9, where the corrected phase shows a stable behavior, exempt of the slow varying drift patterns present in the acquired raw data. On the quantitative analysis, data from experiment draws a phase rms value of $4.5 \mathrm{deg}$, while the corrected plot has a rms value of $2.6 \mathrm{deg}$. When the 3 first hours are removed from this statistical analysis, the rms figure in the corrected phase lowers down to $1.5 \mathrm{deg} \mathrm{rms}$, which can be considered a good accuracy floor integrating the rest of drift sources.

\section{CONCLUSIONS}

An exercise of CATR modeling and compensation has been performed. The complete process was documented, parting from theoretical analysis towards a real case compensation. The proposed approach is cost-effective in the sense that the number of data loggers is reduced to the minimum through the pressure retrieval scheme, being those mass produced inexpensive devices. Quantitative analysis of the model draws excellent performance figures. Future work consists on the joint use of the proposed technique together with quiet zone probing campaigns at millimeter wavelengths so atmospheric compensation is performed.

\section{ACKNOWLEDGMENT}

The first author wishes to thank the former Spanish Ministry of Science and Innovation for the support provided through the FPI scholarship, which made possible the scientific collaboration between ESA-ESTEC and UPM.

\section{REFERENCES}

[1] Olver, A.D.; "Compact antenna test ranges," Antennas and Propagation, 1991. ICAP 91., Seventh International Conference on (IEE), vol., no., pp.99-108 vol.1, 15-18 Apr 1991.

[2] Hirvonen, T.; Ala-Laurinaho, J.; Piironen, P.; Tuovinen, J.; Räisänen, A.V., "A $119 \mathrm{GHz}$ CATR based on a 2.4 Hologram," XXXIII Annual AMTA Symposium, Boston, Massachusetts, November 17-22, 1997.

[3] Muñoz-Acevedo, A.; "Quiet Zone assessment of the CA TR at mmwave frequencies", TEC- EEA/2011.755/MP ESA stay final report.

[4] Vaaja, M.; Häkli, J.; Mallat, J.; Räisänen, A. V.; "Assessment of a Planar Near-Field Range for Quiet-Zone Measurements at $650 \mathrm{GHz}$," XXIX Annual AMTA Symposium, St. Louis, Missouri, 3-8 November 2007.

[5] Saily, J.; Eskelinen, P.; Raisanen, A.V.; "Pilot signal-based real-time measurement and correction of phase errors caused by microwave cable flexing in planar near-field tests," Antennas and Propagation, IEEE Transactions on, vol.51, no.2, pp. 195- 200, Feb 2003.

[6] Liebe, H.J.; Hufford, G.A.; Cotton, M.G.; "Propagation modeling of moist air and suspended water/ice particles at frequencies below 1000 GHz," Proc. NATO/AGARD Wave Propagation Panel, 52nd meeting, No. 3/1-10, Mallorca, Spain, 17 - 20 May, 1993.

[7] UIT-REC-P.453-9-200304

[8] Phillips, W.E.; "The Permittivity of Air at a Wavelength of 10 Centimeters," Proceedings of the IRE, vol.38, no.7, pp. 786- 790, July 1950.

[9] Wayne, R. P. Chemistry of Atmospheres. Oxford University Press, 2000.

[10] Seinfeld, J. H.; Pandis, S. N.; Atmospheric chemistry and physics, J. Wiley, 1998.

[11] Buck, A. L.; "New equations for computing vapor pressure and enhancement factor, “ J. Appl. Meteorol., 20, 1527-1532, 1981.

[12] Yaghjian, A.; "An overview of near-field antenna measurements," Antennas and Propagation, IEEE Transactions on, vol.34, no.1, pp. 30- 45, Jan 1986. 